\title{
Condiciones de la fauna silvestre de tráfico ilegal en un centro de rescate en la ciudad de Guayaquil, Ecuador
}

\author{
Illegal trafficking conditions of wildlife animals in a rescue center in Guayaquil city \\ Romina Richards ${ }^{1}$, María Fernanda Emen ${ }^{2,4}$, Octavio Rugel G. ${ }^{3}$
}

\section{Resumen}

\begin{abstract}
El objetivo del estudio fue identificar las especies afectadas por el tráfico ilegal de fauna silvestre receptadas en un centro de rescate en la ciudad de Guayaquil. Se recopilaron los registros del 2018 al 2020, obteniendo datos de procedencia de rescate, presencia y gravedad de lesiones, y el porcentaje de mortalidad. Adicionalmente, con la identificación del ejemplar, se categorizó según la especie, y su situación de acuerdo con la lista roja de especies amenazadas de la Unión Internacional para la Conservación de la Naturaleza (UICN) y los libros rojos del Ecuador. Se registraron 743 animales de 106 especies, siendo el estadio juvenil el más frecuente. Los animales fueron acogidos por decomiso y entregas voluntarias. Se encontró $10 \%$ de mortalidad, mayormente en mamíferos. De acuerdo con la UICN, la mayoría de los ejemplares se encontraban en la categoría de Preocupación menor, y según los libros rojos del Ecuador, las aves y mamíferos estuvieron situadas en los de Preocupación menor y los reptiles se registraron como Vulnerables en mayor proporción.
\end{abstract}

Palabras clave: fauna, silvestre, conservación, biodiversidad

\footnotetext{
${ }^{1}$ Universidad Agraria del Ecuador, Guayaquil, Ecuador

${ }^{2}$ Departamento de Patología, Facultad de Medicina Veterinaria y Zootecnia, Universidad Agraria del Ecuador, Guayaquil, Ecuador

${ }^{3}$ Departamento de Básico, Facultad de Medicina Veterinaria y Zootecnia, Universidad Agraria del Ecuador, Guayaquil, Ecuador

${ }^{4}$ E-mail:memen@uagraria.edu.ec
}

Recibido: 14 de mayo de 2021

Aceptado para publicación: 12 de noviembre de 2021

Publicado: 22 de diciembre de 2021

CLos autores. Este artículo es publicado por la Rev Inv Vet Perú de la Facultad de Medicina Veterinaria, Universidad Nacional Mayor de San Marcos. Este es un artículo de acceso abierto, distribuido bajo los términos de la licencia Creative Commons Atribución 4.0 Internacional (CC BY 4.0) [https:// creativecommons.org/licenses/by/4.0/deed.es] que permite el uso, distribución y reproducción en cualquier medio, siempre que la obra original sea debidamente citada de su fuente original 
The aim of this study was to identify the species affected by the illegal trafficking of wildlife received in a rescue centre in the city of Guayaquil. Records from 2018 to 2020 were collected, obtaining data on the origin of rescue, presence and severity of injuries, and the percentage of mortality. Additionally, with the identification of the specimen, it was categorized according to the species, and its situation according to the red list of threatened species of the International Union for the Conservation of Nature (IUCN) and the red books of Ecuador. In total, 743 animals of 106 species were registered, the juvenile stage being the most frequent. The animals were taken in by confiscation and voluntary surrender. Ten percent mortality was found, mostly in mammals. According to the IUCN, most of the specimens were in the category of Least Concern, and according to the red books of Ecuador, birds and mammals were classified in those of Least Concern and reptiles were recorded as Vulnerable in a higher proportion.

Key words: fauna, wild, conservation, biodiversity

\section{INTRODUCCIÓN}

La disminución de la biodiversidad es uno de los problemas más graves desde el punto de vista ambiental y de ecosistemas, el cual se ha incrementado con el paso de los años por factores antropogénicos tales como destrucción de hábitats, cambio climático y la comercialización de especies de vida silvestre (Travis, 2003). Las altas tasas de extinción de las especies generan un gran impacto en la biodiversidad de los ecosistemas (Ceballos et al., 2015).

La extinción se ha visto equilibrada con la especiación; sin embargo, se han registrado cinco grandes extinciones masivas denominadas así, por la extensión en los picos de pérdidas de más del $75 \%$ de especie (Ritchie y Roser, s.f.). Se plantea que la sexta extinción masiva está en marcha debido a que la tasa de extinción es casi cien veces mayor a la esperada, afectando a mamíferos, anfibios y aves (Barnosky et al., 2011).

El tráfico de animales de fauna silvestre es una de las actividades ilegales más lucrativas, generando ganancias globales esti- madas entre 8 y 10 mil millones de dólares al año (Lawson y Vines, 2014). Las repercusiones de la extracción de un animal silvestre de su hábitat natural implican un desequilibrio en el ecosistema y ruptura de la cadena biológica, ya que este espécimen pierde la oportunidad de reproducirse y contribuir a la perpetuación de su especie. En muchas ocasiones, al ser sustraída una madre las crías pueden no sobrevivir sin el cuidado de ella (Mancera y Reyes, 2008).

El Ecuador a pesar de contar con una pequeña extensión de superficie, es considerado uno de los países con mayor diversidad, tanto en la flora como la fauna (Roy et al., 2018). En el país, una de las zonas más afectadas por esta actividad ilegal es la Amazonia debido a la gran variedad de especies que en ella habitan. La constitución y leyes ecuatorianas prohíben la comercialización y tenencia de la fauna silvestre, pero no impiden la caza de subsistencia, por lo que algunas comunidades indígenas se dedican a la caza para consumo familiar y a la venta de partes para artesanías y rituales en su cultura (Asamblea Nacional República del Ecuador, 2017). 
La historia de la cacería con fines comerciales en la provincia del Guayas no está bien documentada. Ante esto, el propósito del presente estudio fue recopilar los registros del Proyecto Sacha, el cual funciona como centro de paso de vida silvestre acerca de las especies afectadas, motivos y lugares de extracción o decomiso, facilitando una línea base de base que ayude a la elaboración de líneas estratégicas en los planes de conservación de las especies que, además de ser traficadas, se encuentran en alguna categoría de amenaza que pueda afectar la población de estas a través del tiempo.

\section{Materiales y Métodos}

La investigación fue cuantitativa de tipo descriptiva, diseño no experimental y de corte transversal. Se realizó en el Centro de Rescate Proyecto Sacha, ubicado en la ciudad de Guayaquil, provincia del Guayas, Ecuador. Los registros de los animales de vida silvestre que llegaron al centro corresponden al periodo entre octubre de 2018 a enero de 2020.

Para determinar la gravedad de las lesiones de los individuos registrados, se utilizó la Escala Abreviada de Lesiones (AIS, por sus siglas en inglés). Esta se utiliza principalmente en casos agudos y de atención médica para clasificar a los pacientes y planificar los diferentes niveles de atención necesarios según las distribuciones de gravedad (Robertson, 2018). Las lesiones fueron clasificadas por sistemas corporales, dividiendo al cuerpo en seis regiones: (1) cabeza y cuello, (2) cara, (3) tórax, (4) abdomen, (5) extremidades, y (6) cinturón escapular y pelviano (Rapsang y Shyam, 2015). Los registros recopilados se procesaron en una hoja electrónica de Microsoft Excel mediante tablas de frecuencia.

\section{Resultados y Discusión}

Se registraron 743 animales de 106 especies en el Proyecto Sacha en el periodo indicado (Cuadro 1). De estos, 27 especies representan el $75 \%$ del total de animales receptados, siendo la zarigüeya Didelphis marsupialis y la tortuga charapa arrau Podocnemis expansa las dos especies con mayor representatividad.

El 49\% de los animales receptados correspondieron a jóvenes, $36 \%$ a adultos y $16 \%$ a neonatos. Por otro lado, la mayor proporción de animales provinieron de zonas urbanas, mientras que $18 \%$ provinieron de zonas rurales de la provincia del Guayas.

La Figura 1 muestra las proporciones de animales que se retuvieron por decomiso o por entrega voluntaria. La mayor proporción de reptiles fueron entregados voluntariamente, mientras que en el caso de mamíferos y aves estos tuvieron que ser decomisados. Se entiende por «Decomiso» los animales que las autoridades confiscan y entregan al Proyecto Sacha en tanto que «Entrega Voluntaria»s se refiere a los animales cuyos «dueños» los entregan de manera voluntaria. Entre los animales recibidos se encontraban representantes de 45 especies $(42.45 \%)$ relacionadas directamente con el tráfico y tenencia ilegal, de las cuales 15 fueron aves (33.3\%) con el Brotogeris versicolurus como representante principal, 19 mamíferos (42.2\%) con el Saimiri sciureus en primer lugar y 11 reptiles (24.4\%) encabezados por Podocnemis expansa.

Estos datos difieren con Ordoñez (2012), quien identificó que las aves obtenían un primer lugar con un $71 \%$, seguido de reptiles con $16 \%$ y por último mamíferos con $13 \%$ en la provincia de Orellana- Ecuador, y con el estudio realizado por Bazurto (2018), 
Cuadro 1. Especies más frecuentes de fauna silvestre receptadas a través del Proyecto Sacha entre octubre de 2018 a enero de 2020

\begin{tabular}{llcc}
\hline Grupo & Especie & $\mathrm{n}$ & $\%$ \\
\hline Mamífero & Didelphis marsupialis & 118 & 16 \\
Reptil & Podocnemis expansa & 104 & 14 \\
Ave & Tyto alba & 32 & 4 \\
Mamífero & Leopardus pardalis & 28 & 4 \\
Ave & Psittacara erythrogenys & 20 & 3 \\
Ave & Brotogeris pyrrhoptera & 19 & 3 \\
Reptil & Iguana iguana & 16 & 2 \\
Reptil & Kinosternon scorpioides & 16 & 2 \\
Ave & Forpus coelestis & 15 & 2 \\
Reptil & Podocnemis unifilis & 15 & 2 \\
Mamífero & Saimiri sciureus & 15 & 2 \\
Ave & Pionus menstruus & 14 & 2 \\
Reptil & Boa constrictor & 13 & 2 \\
Ave & Dendrocygna bicolor & 13 & 2 \\
Ave & Brotogeris versicolurus & 12 & 2 \\
Mamífero & Choloepus hoffmanni & 12 & 2 \\
Ave & Glaucidium peruanum & 12 & 2 \\
Ave & Amazilia amazilia & 11 & 1 \\
Mamífero & Procyon cancrivorus & 11 & 1 \\
Ave & Glaucidium nubicola & 10 & 1 \\
Mamífero & Sciurus stramineus & 9 & 1 \\
Mamífero & Alouatta palliata & 8 & 1 \\
Ave & Rostrhamus sociabilis & 8 & 1 \\
Reptil & Chelydra acuirostris & 7 & 1 \\
Mamífero & Herpailurus yagouaroundi & 7 & 1 \\
Reptil & Rhinoclemmys melanosterna & 7 & 1 \\
Mamífero & Tamandua mexicana & 7 & 2 \\
\hline & & & 1 \\
\hline
\end{tabular}

quien identificó igualmente que las aves en Manglares Churute (Ecuador) eran las más afectadas. No obstante, el Ministerio del Ambiente del Ecuador (2013), quien identificó que los mamíferos son el grupo más afectado (32\%), seguido de aves (25\%), reptiles $(23 \%)$ y por último peces $(20 \%)$.
Las especies relacionadas al tráfico ilegal fueron registradas mayormente en la provincia del Guayas: 13 especies de aves (86.7\%), 16 especies de mamíferos (84.2\%) y 9 especies de reptiles (81.8\%); seguido se la provincia de Los Ríos y de la Provincia de Santa Elena, aunque hubo varios casos don- 


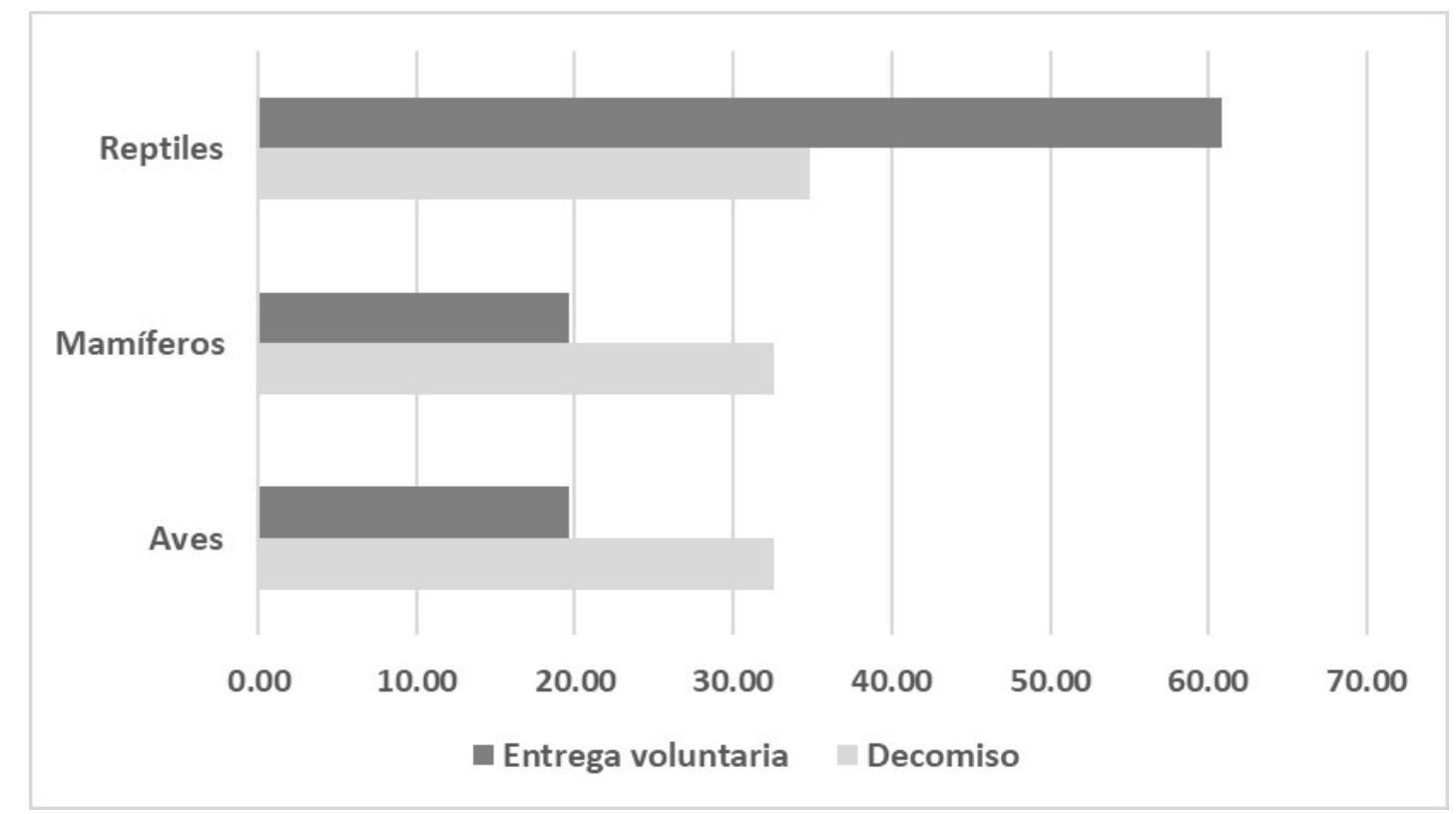

Figura 1. Frecuencia de los motivos de retención del animal por tipo de especie en el Proyecto Sacha entre octubre de 2018 a enero de 2020

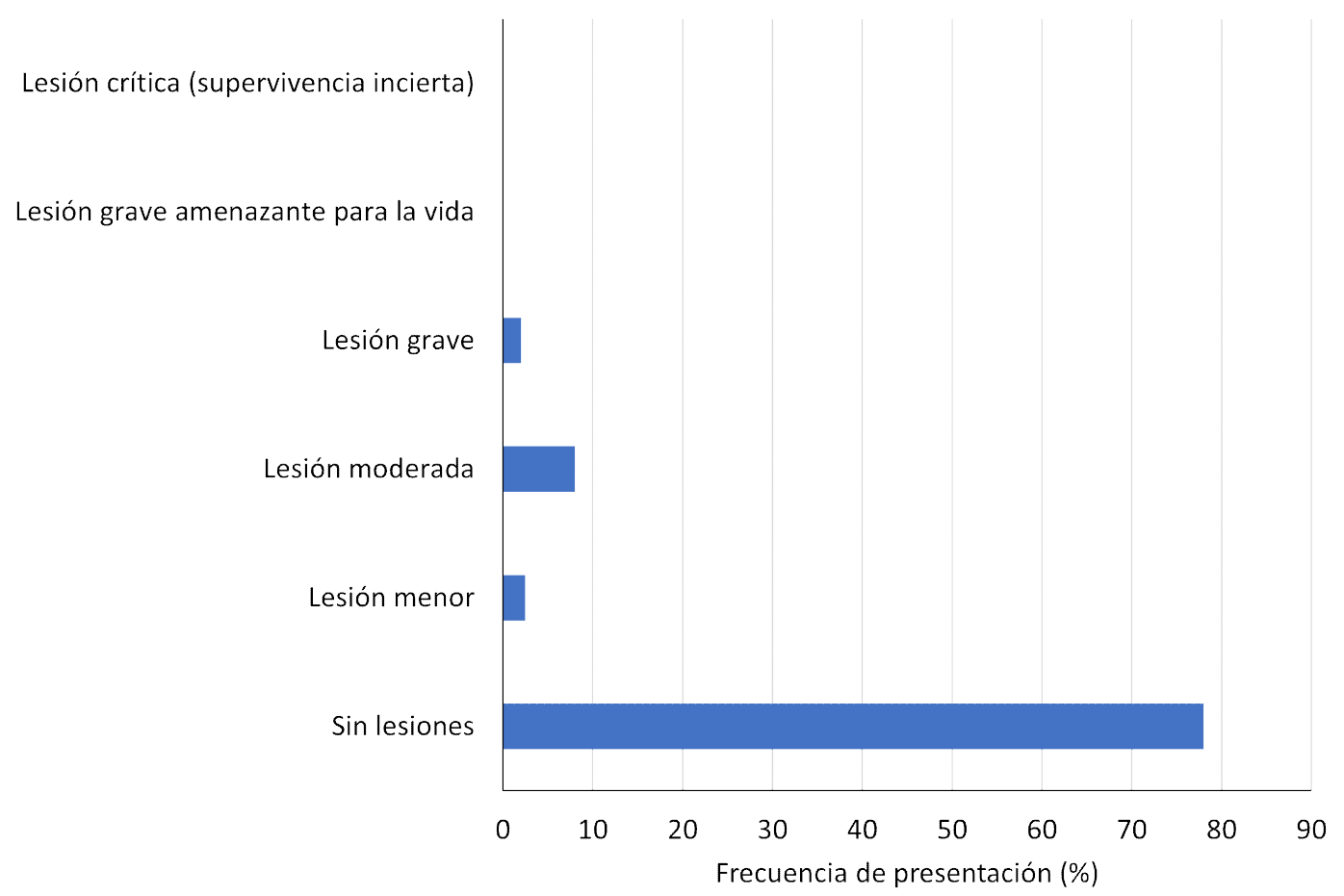

Figura 2. Gravedad de lesiones de animales receptados en el proyecto Sacha en el periodo octubre de 2018 a enero de 2020 
Cuadro 2. Nivel de lesiones en animales acogidos en el Proyecto Sacha entre octubre de 2018 a enero de 2020

\begin{tabular}{lccc}
\hline & Aves & Mamíferos & Reptiles \\
\hline $\begin{array}{l}\text { Sin } \\
\text { lesiones }\end{array}$ & 92.5 & 70 & 95.2 \\
$\begin{array}{l}\text { Lesiones } \\
\text { menores }\end{array}$ & 7.5 & 27.5 & 3.2 \\
$\begin{array}{l}\text { Lesiones } \\
\text { graves }\end{array}$ & 0 & 2.5 & 1.6 \\
\hline
\end{tabular}

de no se registró la procedencia. Bazurto (2018), igualmente encontró que la mayoría de los procesos registrados provenían de diferentes cantones de la provincia del Guayas $(90 \%)$.

El 78\% de los animales receptados no presentó lesiones corporales y solo $2 \%$ presentó lesiones graves (Figura 2). La frecuencia del nivel de lesiones para las aves, mamíferos y reptiles se muestra en el Cuadro 2. No se disponen de datos similares en la literatura leída de modo que no se pudo contrastar estos datos con otros similares.

La mortalidad general fue de $9.79 \%$. Las aves registraron $15 \%$, los mamíferos $17.5 \%$ y los reptiles $1.6 \%$, resultados similares a los reportados por Morocho y Reyes (2012) para la zona de Zamora Chinchipe (Ecuador), donde la mortalidad en mamíferos fue de $49.6 \%$, en aves de $38.4 \%$ y en reptiles de $12.1 \%$

Se encontraron seis categorías en la clasificación de especies afectadas por el tráfico y tenencia ilegal registradas en Proyecto Sacha según la Unión Internacional para la Conservación de la Naturaleza (https:// www.iucn.org/es). De estas, 30 especies (66.7\%) como se encontraban en la categoría de Preocupación Menor, 4 especies (8.9\%) como Casi Amenazada, 5 especies (11.1\%) como Vulnerable, 1 especie (2.2\%) En Peli- gro, 1 especie (2.2\%) En Peligro Crítico y 4 especies (8.9\%) como No evaluada. Orlando (2019), por su parte, reportó para la provincia de Manabí, Ecuador, en el periodo 20162018 que $64 \%$ de las especies pertenecían a Preocupación Menor, 21\% a categoría Vulnerable y $15 \%$ En Peligro, concordando en que la mayoría de las especies que se registran por tráfico y tenencia ilegal se encuentran categorizadas como Preocupación menor.

Según el Libro Rojo de Aves del Ecuador, se encontraron especies de aves de vida silvestre afectadas por el tráfico y tenencia ilegal en cinco categorías: Preocupación Menor (66.67\%), Casi Amenazadas (13.33\%), Vulnerable (6.67\%), En Peligro (6.67\%) y No Evaluada (6.67\%). Felicita (2019) menciona que los Psittacidos fueron el grupo más representativo en los centros de manejo de fauna silvestre, coincidiendo con este estudio donde se identificó a Brotogeris versicolurus, ave Psittacida.

Las especies de mamíferos de vida silvestre afectadas por el tráfico y tenencia ilegal, según el Libro Rojo de Mamíferos del Ecuador se clasificaron en Preocupación Menor (26.32\%), Casi Amenazadas (15.79\%), Vulnerables (26.32\%), En Peligro (15.79\%), en Peligro Crítico (5.26\%) y Datos Insuficientes (5.26\%). Además, se presentó una especie de mamífero exótico. Ordoñez (2012), por su parte, encontró que $67 \%$ de los mamíferos registrados en su estudio pertenecen a la categoría de Preocupación Menor y 33\% a la categoría Vulnerable. Asimismo, las especies Leopardus pardalis, Saimiri sciureus y Alouatta palliata coinciden con las del estudio de Felicita (2019) como las especies de mayor frecuencia.

En cuanto a los reptiles de vida silvestre afectadas por el tráfico y tenencia ilegal, de acuerdo con el Libro Rojo de Reptiles del Ecuador se clasificaron en seis categorías: Vulnerables (36.36\%), En Peligro (27.27\%), Preocupación Menor (9.09\%), Casi Amena- 
zada (9.09\%), En Peligro Crítico (9.09\%) y Extranjero (9.09\%). Ordoñez (2012) encontró que las especies catalogadas en Preocupación Menor, Vulnerable y En Peligro Crítico se encontraron en igual proporción.

\section{Agradecimiento}

Al Proyecto Sacha por su labor en el rescate y conservación de las especies de vida silvestre y por su apertura a los registros durante la fase de investigación

\section{Literatura Citada}

1. Asamblea Nacional República del Ecuador. 2017. Código Orgánico del Ambiente. [Internet]. Disponible en: https://www.asambleanacional.gob.ec/ sites/default/files/private/asambleanacional/filesasambleanacionalnameuid-29/ Leyes\%202013-2017/102-ambiente/rocod-ambiente-ro-s-983-12-04-2017.pdf

2. Barnosky AD, Matzke N, Tomiya S, Wogan GO, Swartz B, Quental TB, Marshall C, et al. 2011. Has the Earth's sixth mass extinction already arrive? Nature 471: 51-57. doi:10.1038/nature09678

3. Bazurto K. 2018. Tráfico de Fauna Silvestre en la Reserva Ecológica Manglares Churute 2015-2017. Tesis de Biólogo. Guayaquil, Ecuador: Univ. de Guayaquil. $49 \mathrm{p}$.

4. Ceballos G. Ehrlich PR, Barnosky AD, García A, Pringle RM, Palmer TM. 2015. Accelerated modern humaninduced species losses: entering the sixth mass extinction. Sci Adv 1(5): e1400253. doi: 10.1126/sciadv.1400253

5. Felicita E. 2019. Evaluación de las dietas para aves y mamíferos de los principales centros de manejo de fauna del Ecuador y diseño de una herramienta informática para valoración de la nutrición de fauna en cautiverio. Tesis de Ingeniería en Gestión Ambiental. Quito, Ecuador: Univ. Central del Ecuador. 68 p.
6. Lawson K, Vines A. 2014. Global impacts of the illegal wildlife trade. The costs of crime, insecurity and institutional erosion. London, UK: Chatham House. 50 p.

7. Mancera N, Reyes O. 2008. Comercio de fauna silvestre en Colombia. Rev Fac Nac Agron Medellín 61: 4618- 4645.

8. Ministerio del Ambiente. 2013. Informe del tráfico ilegal de especies en el Ecuador Continental en el año 2013. [Internet]. Disponible en: http://suiadoc.ambiente.gob.ec/documents/10179/ 346527 / In forme +de + tr\% C3\%A1fico+ilegal+2013.pdf/eb6c31414f34-4cf5-b138-710575667aab?version $=1.0$

9. Morocho D, Reyes M. 2012. Estudio del tráfico ilegal de fauna silvestre en la provincia de Zamora Chinchipe. Tesis de Ingeniería en Gestión Ambiental. Loja, Ecuador: Univ. Técnica Particular de Loja. $162 \mathrm{p}$.

10. Rapsang AG, Shyam DC. 2015. Compendio de las escalas de evaluación de riesgo en el paciente politraumatizado. Cirugía Española 93: 213-221. doi: 10.1016/j.ciresp.2013.12.021

11. Ritchie H, Roser M. s.f. Our World in Data. [Internet]. Available in: https:// ourworldindata.org/extinctions\#the-bigfive-mass-extinctions

12. Robertson LS. 2018. Injury epidemiology. $4^{\text {th }}$ ed. Lulu Books. 364 p.

13. Roy BA, Zorrilla M, Endara L, Thomas DC, Vandegrift R, Rubenstein JM, Policha T, et al. 2018. New mining concessions could severely decrease biodiversity and ecosystem services in Ecuador. Trop Conserv Sci 11: 1 - 20. doi: 10.1177/1940082918780427

14. Ordoñez A. 2012. Estudio del tráfico ilegal de especies de fauna silvestre en la provincia de Orellana. Tesis de Ingeniería en Gestión Ambiental. Loja, Ecuador: Univ. Técnica Particular de Loja. 130 p.

15. Orlando D. 2019. Análisis del tráfico ilegal de vida silvestre en la provincia de Manabí. Tesis de Ingeniero en Medio Ambiente. Ecuador: Univ. Estatal del Sur de Manabí. 102 p. 
16. Soler R, Benitez J, Goyenechea G, Ramos $S$, Albertini G, Ramírez A, García J. 2010. Evaluación pronóstica en el traumatizado. La Habana, Cuba. 55 p.

17. Travis JM. 2003. Climate change and habitat destruction: a deadly antropo- genic cocktail. Proc Biol Sci 270: 467473. doi:10.1098/rspb.2002.2246

18. [UICN] Unión Internacional para la Conservación de la Naturaleza. $(08$ de Noviembre de 2021). UICN. Obtenido de UICN: https://www.iucn.org/es 